\title{
Stochastic and random models of Malaria Disease with vertical transmission
}

\author{
Zafer Bekiryazici ${ }^{3 *}$, Tulay Kesemen ${ }^{2}$,Mehmet Merdan ${ }^{1}$ \\ ${ }^{1}$ Department of Mathematical Engineering, Gumushane University, Gumushane, Turkey \\ ${ }^{2}$ Department of Mathematics, Karadeniz Technical University, Trabzon, Turkey \\ ${ }^{3}$ Department of Mathematics, Recep Tayyip Erdogan University, Rize, Turkey
}

\begin{abstract}
Malaria is an infectious disease which affects both humans and animals. In this study, the existing mathematical model of malaria disease with vertical transmission is analyzed in random enviroment. Random effect terms are added to the parameters of the deterministic model to form a system of random differential equations. Similarly, stochastic noise is added to the deterministic system to obtain a stochastic model. Finally, the results from the deterministic, random and stochastic model are compared to comment on the random behavior of the disease.
\end{abstract}

Keywords: Malaria, stochastic differential equation, random differential equation, Milstein method, normal distribution.

\section{Introduction}

Mathematical models, which are equations systems expressing real life events, are widely used in biology, engineering, health and many areas of social sciences. Analysis of models provide a wider perspective for the events under consideration. Models of epidemiological diseases offer guidance to studies for battling the diseases and shape many countries health policy. Most of the mathematical modeling studies in the area of health, medicine and biology are carried out on a deterministic level. However, deterministic events produce the same results under the same conditions and it is known that most of the parameters used in mathematical models of health and biology are open to variations in different trials. Thus, a probabilistic modeling approach is more suitable for these areas. Diseases can be modeled on a probabilistic level using stochastic noise or random effect terms [4].

Malaria is a mosquito-borne infectious disease. Fever, headaches and vomiting are amongst the typical sypmtoms of the disease. The infection is caused by a species of Plasmodium which is a parasite transmitted by an infected mosquito of the Anopheles type [6]. One type of parasite causes malaria infections which can cause death in some cases, while other 3 types of the parasite cause a mild infection [5],[15]. A study states that Malaria kills between 1.1-2.7 million people every year, which includes about a million children under five in Africa [8]. Latest reports from World Health Organization (WHO) estimate that there were 214 million new infections of malaria worldwide in 2015, where $88 \%$ of the malaria cases are seen in Africa [15].

Several authors have made modeling studies on the dynamics of malaria infection on a deterministic level [1], [2], [3], [5], [6], [13], [16]. While most of the vast literature on the modeling studies of malaria are deterministic studies, several studies examine stochastic aspects of the disease as well [9], [10], [14]. However, a modeling approach which compares 
random and stochastic forms of this model of malaria disease with vertical transmission has not been given. Firstly, the deterministic model which exists in the referred study will be introduced along with the numerical solutions [13]. This model will be used for building random and stochastic models and the results from these models will be given. Finally, the results of all three models will be compared and comments on the random dynamics of malaria will be stated.

\section{Deterministic model of Malaria}

The deterministic model of Malaria with vertical transmission is given by the following system [13].

$$
\begin{aligned}
& \frac{d}{d t} S_{h}=\mu(1-\pi)-\sigma S_{h}-\eta S_{h} I_{v}+k I_{h}+\left(1-I_{h}\right) \varphi \\
& \frac{d}{d t} I_{h}=\eta S_{h} I_{v}-(k+m) I_{h} \\
& \frac{d}{d t} I_{v}=I_{h}\left(1-I_{v}\right)-\omega I_{v}
\end{aligned}
$$

The variables in the equation system are $S_{h}, I_{h}$ and $I_{v} . S_{h}$ describes the ratio of the susceptible humans in the total population $N_{v}, I_{h}$ describes the ratio of the infected humans in the total population and $I_{v}$ describes the ratio of the infected mosquitoes in the total vector population. Hence, model (1) is formed by 3 differential equations examining the course of the disease by describing the changes in the ratios of the susceptible humans and infected humans-mosquitoes. Model (1) is obtained in [13] from a system of 5 equations. The parameters of (1) are also derived from the parameters of the original system. $\mu=\frac{\mu_{H}}{a c}$, where $\mu_{H}$ is the birth-death rate of host population, $a$ is the average infection rate on man by a single mosquito and $c$ is the probability that a mosquito becomes infectious. $\pi=\rho s$, where $\rho$ is vaccinated portion of the newborn hosts and $s$ is the effectiveness of the vaccine. $\sigma=\frac{\mu_{H}+\gamma}{a c}$, where $\gamma$ is the per capita loss rate of immunity in hosts. $\eta=\frac{b \phi}{c}$, where $b$ is the proportion of bites that cause an infection on hosts and $\phi$ is the vector-host population ratio. $k=\frac{v}{a c}$, where $v$ is the rate of recovery of hosts. $\varphi=\frac{\gamma}{a c} \cdot m=\frac{\mu_{H}+r}{a c}$, where $r$ is the rate that hosts gain immunity. $\omega=\frac{\mu_{V}}{a c}$, where $\mu_{V}$ is the birth-death rate of vector population.

Numerical solutions of the deterministic model (1) will be compared with the random and stochastic results, since the equations are nonlinear and their exact solutions are complex. The values of the parameters in system (1) and the initial values of the variables are as follows [1], [12], [13].

$\mu=1, \pi=0.005, \sigma=2.1724, \eta=0.00492, \varphi=0.9, k=2, m=1.2, \omega=1, S_{h}(0)=0.5, I_{h}(0)=0.5, I_{v}(0)=0.4$

\subsection{Deterministic results}

The numerical results of the deterministic system are obtained in MATLAB, using the built-in lower order schemes (Figures $1 \& 2$ ). The deterministic behavior of the variables can be seen in a single graph in Figure 1. The graph shows that the ratio of susceptible humans increases fastly in the beginning of the process and keeps a certain level throughout the process. $S_{h}$ gets a minimum value of 0.5 at $t=0$ and a maximum value of 0.874 at $t=1.554$. Both of the ratio of infected humans and infected mosquitoes decrease though the process. $I_{h}$ gets a minimum value of $2.171 \times 10^{-10}$ at $t=15$ and a maximum value of 0.5 at $t=0$, while $I_{v}$ gets its minimum value $1.114 \times 10^{-7}$ at $t=15$ and its maximum value 0.4 at $t=0$. The deterministic results of the variables can be seen in seperate graphs as follows. Note that the time interval $[0,15]$ has been used to make a similar analysis to the referred article [13]. The results show that the infected host and vector population keeps decreasing from the start to the end, meaning that the disease is being removed from the population. 


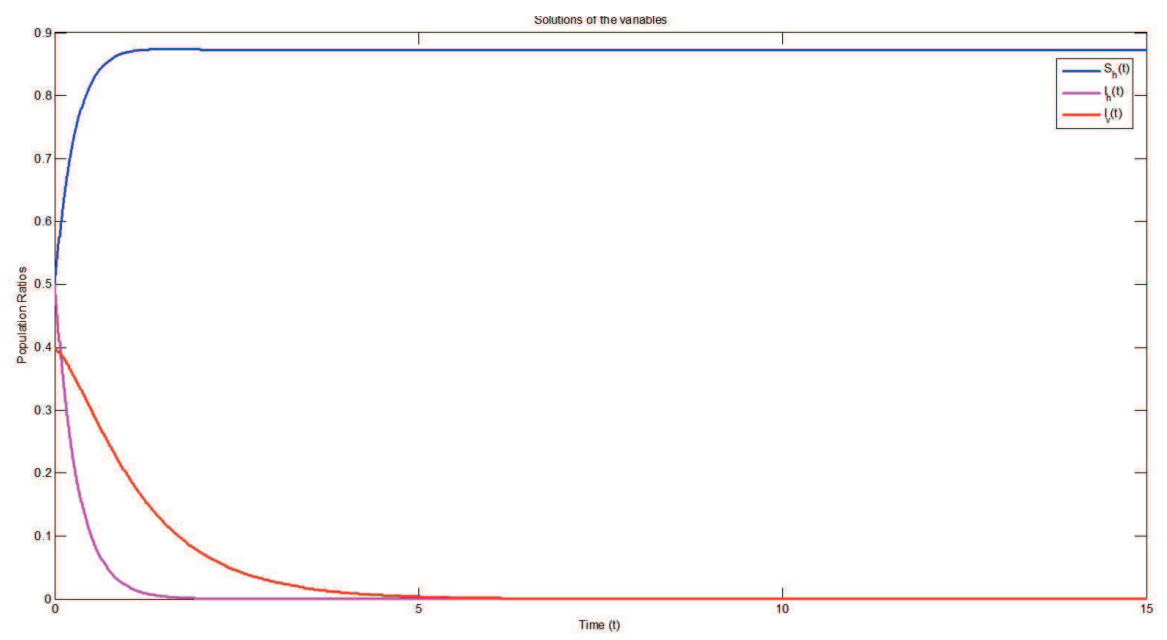

Fig. 1: Deterministic behavior of the variables.
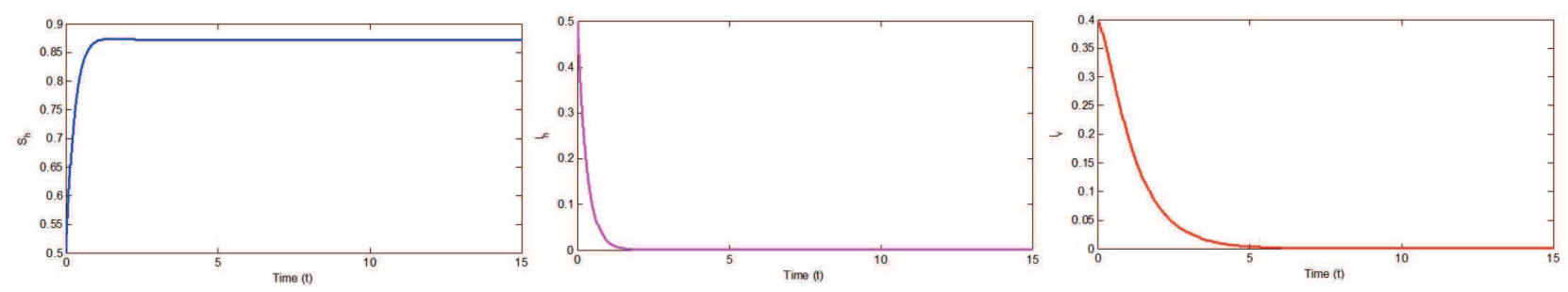

Fig. 2: Deterministic results for $S_{h}, I_{h}$ and $I_{v}$, respectively.

\section{Random model}

The deterministic system (1) will be used to obtain a random model of malaria disease. The values of the parameters of the deterministic model are obtained through statistical studies. The data obtained from patients are analyzed to determine the parameters describing various aspects of the disease. However, since these values are the results of statistical analyses, they are open to variations in real life. Thus, the parameters of system (1) are added random effect terms to obtain a system of random differential equations for modeling malaria under random conditions. The motivations of this analysis are the previous studies from the authors [4],[11].

In probability theory, usually normal distribution is used for parameters for which the exact distribution is unknown, since the distribution of values which are affected by many factors are usually almost normal. Thus, we will be adding random effects with normal distribution (Gaussian) to the parameters of the model. The new set of parameters will become.

$$
\begin{aligned}
& \mu^{*}=\mu_{0}+\delta_{1} \gamma_{1}, \pi^{*}=\pi_{0}+\delta_{2} \gamma_{2}, \sigma^{*}=\sigma_{0}+\delta_{3} \gamma_{3}, \eta^{*}=\eta_{0}+\delta_{4} \gamma_{4}, \\
& \varphi^{*}=\varphi_{0}+\delta_{5} \gamma_{5}, k^{*}=k_{0}+\delta_{6} \gamma_{6}, m^{*}=m_{0}+\delta_{7} \gamma_{7}, \omega^{*}=\omega_{0}+\delta_{8} \gamma_{8},
\end{aligned}
$$

where the zero-indexed parameters $\mu_{0}, \pi_{0}, \sigma_{0}, \eta_{0}, \varphi_{0}, k_{0}, m_{0}, \omega_{0}$, are the original numerical values of the parameters, the coefficients $\delta_{i}, i=\overline{(1,8)}$ are the standard deviations of the new random variables $\mu^{*}, \pi^{*}, \sigma^{*}, \eta^{*}, \varphi^{*}, k^{*}, m^{*}, \omega^{*}$ and $\gamma_{i}, i=\overline{(1,8)}$ are independent and identically distributed random variables with standard normal distribution. The exact distributions of the parameters are unknown, so the standard deviations of the random parameters are determined to be 
around $5 \%$ of their mean values, which are accepted to be $\mu_{0}, \pi_{0}, \sigma_{0}, \eta_{0}, \varphi_{0}, k_{0}, m_{0}, \omega_{0}$. Thus, the set of random parameters are given as.

$$
\begin{aligned}
& \mu^{*}=1+0.05 \gamma_{1}, \pi^{*}=0.005+0.00025 \gamma_{2}, \sigma^{*}=2.1724+0.10862 \gamma_{3}, \eta^{*}=0.00492+2.46 \times 10^{-4} \gamma_{4}, \\
& \varphi^{*}=0.9+0.045 \gamma_{5}, k^{*}=2+0.1 \gamma_{6}, m^{*}=1.2+0.06 \gamma_{7}, \omega^{*}=1+0.05 \gamma_{8},
\end{aligned}
$$

Using the random parameters above, the random model of malaria with vertical transmission which consists of differential equations describing the random behavior of the parameters $S_{h}, I_{h}$ and $I_{v}$ becomes.

$$
\begin{aligned}
\frac{d}{d t} S_{h}= & \left(1+0.05 \gamma_{1}\right)\left(1-\left(0.005+0.00025 \gamma_{2}\right)\right)-\left(2.1724+0.10862 \gamma_{3}\right) S_{h}- \\
& \left(0.00492+2.46 \times 10^{-4} \gamma_{4}\right) S_{h} I_{v}+\left(2+0.1 \gamma_{6}\right) I_{h}+\left(1-I_{h}\right)\left(0.9+0.045 \gamma_{5}\right) \\
\frac{d}{d t} I_{h}= & \left(0.00492+2.46 \times 10^{-4} \gamma_{4}\right) S_{h} I_{v}-\left(\left(2+0.1 \gamma_{6}\right)+\left(1.2+0.06 \gamma_{7}\right)\right) I_{h} \\
\frac{d}{d t} I_{v}= & I_{h}\left(1-I_{v}\right)-\left(1+0.05 \gamma_{8}\right) I_{v}
\end{aligned}
$$

along with the same initial conditions: $S_{h}(0)=0.5, I_{h}(0)=0.5, I_{v}(0)=0.4$.

\subsection{Random results}

The results for the random model (2) are obtained by simulating the system in MATLAB.

\subsubsection{Expected values}

The expectations can be given in a single graph for a comparison with the deterministic results of model (1) as below (Figure 3). Maximum and minimum values of expected values of the random variables are obtained as follows: $S_{h}$ takes

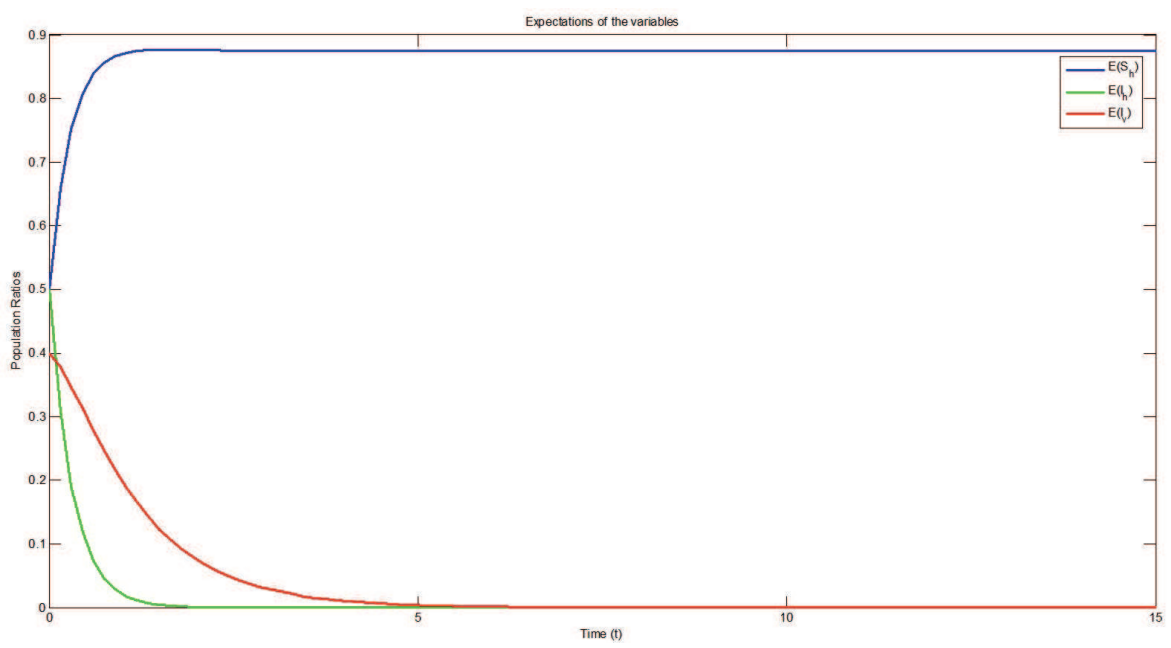

Fig. 3: Expectations of the random variables.

its maximum value 0.8764 at $t=1.65$ and its minimum value 0.5 at $t=0 . I_{h}$ gets it maximum value 0.5 at $t=0$ and its minimum value $4.495 \times 10^{-10}$ at $t=15$, while $I_{v}$ gets its maximum and minimum values 0.4 and $2.324 \times 10^{-7}$ at $t=0$ 
and $t=15$, respectively. It can be seen that the results for $S_{h}, I_{h}$ and $I_{v}$ are very similar with their deterministic counterparts.

The expected values of $S_{h}, I_{h}$ and $I_{v}$ are given below (Figure 4).
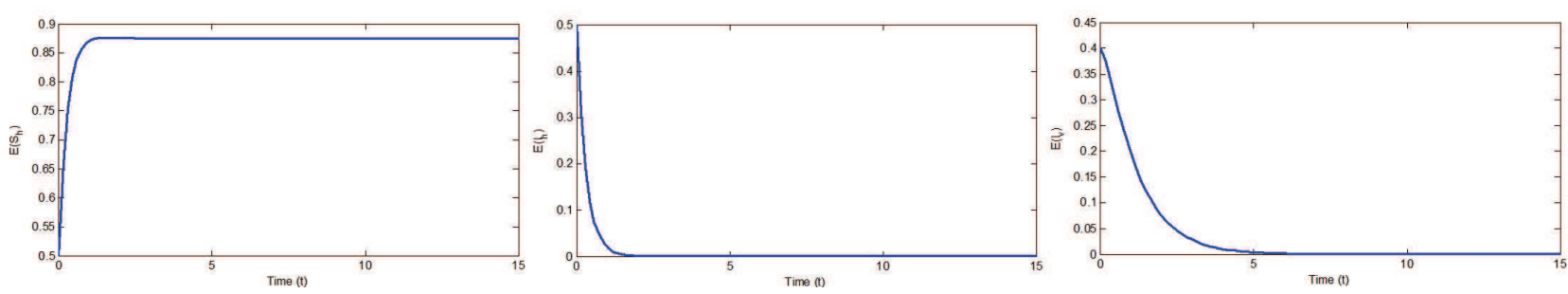

Fig. 4: The expectations of the variables $S_{h}, I_{h}$ and $I_{v}$, respectively.

\subsubsection{Variances}

The variances of $S_{h}, I_{h}$ and $I_{v}$ are given below (Figure 5). Extremum values of the variances of the random variables are
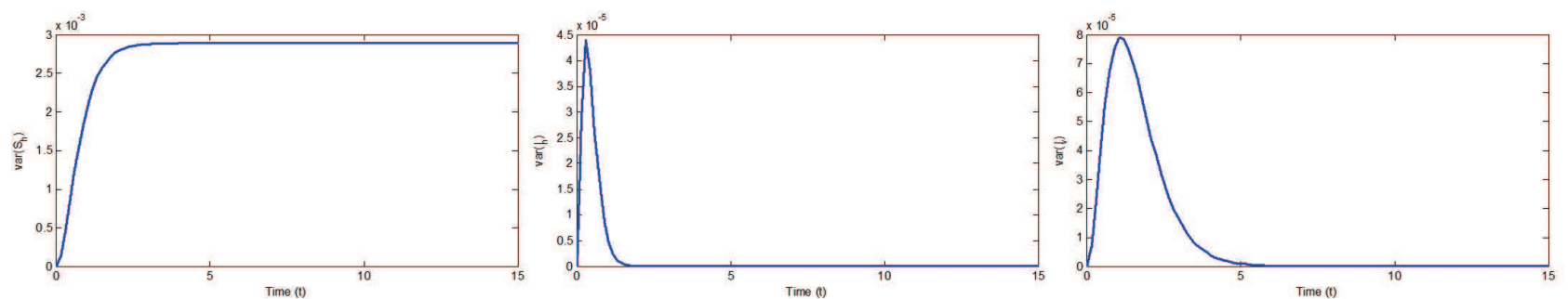

Fig. 5: Variances of the variables $S_{h}, I_{h}$ and $I_{v}$, respectively.

obtained as follows: $\min \left[\operatorname{var}\left(S_{h}\right)\right]=0$ at $t=0$ and $\max \left[\operatorname{var}\left(S_{h}\right)\right]=0.002896$ at $t=15 . \min \left[\operatorname{var}\left(I_{h}\right)\right]=0$ at $t=0$ and $\max \left[\operatorname{var}\left(I_{h}\right)\right]=4.41 \times 10^{-5}$ at $t=0.3 . \min \left[\operatorname{var}\left(I_{v}\right)\right]=4.033 \times 10^{-27}$ at $t=0$ and $\min \left[\operatorname{var}\left(I_{v}\right)\right]=7.879 \times 10^{-5}$ at $t=1.05$.
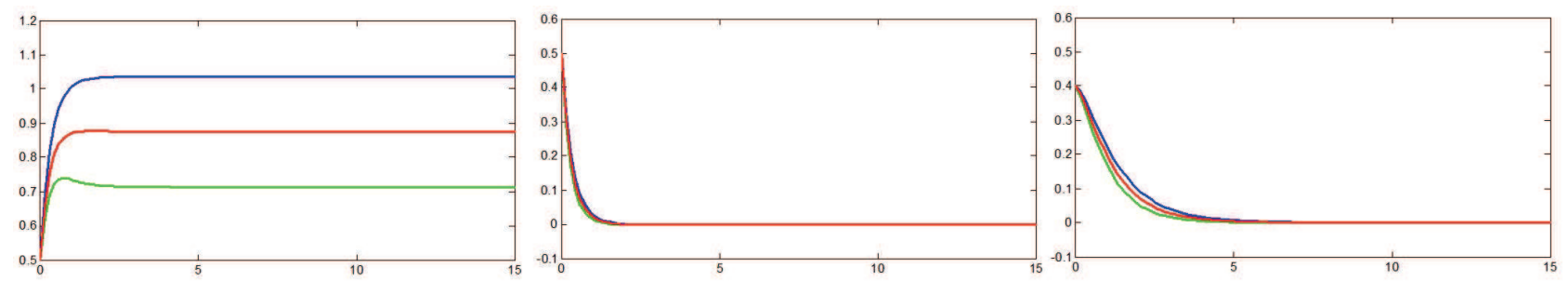

Fig. 6: Confidence intervals of the variables $S_{h}, I_{h}$ and $I_{v}$, respectively. 


\subsubsection{Confidence intervals}

The confidence intervals of $S_{h}, I_{h}$ and $I_{v}$ are given in Figure 6. The extremum values of the confidence intervals are as follows: $\min \left(E\left(S_{h}\right) \mp K \sqrt{\operatorname{var}\left(S_{h}\right)}\right)=0.5$ at $t=0$ and $\max \left(E\left(S_{h}\right) \mp K \sqrt{\operatorname{var}\left(S_{h}\right)}\right)=1.037$ at $t=15 . \min \left(E\left(I_{h}\right) \mp\right.$ $\left.K \sqrt{\operatorname{var}\left(I_{h}\right)}\right)=-7.773 \times 10^{-8}$ at $t=0$ and $\max \left(E\left(I_{h}\right) \mp K \sqrt{\operatorname{var}\left(I_{h}\right)}\right)=0.5$ at $t=0 . \min \left(E\left(I_{v}\right) \mp K \sqrt{\operatorname{var}\left(I_{v}\right)}\right)=-4.481 \times$ $10^{-5}$ at $t=7.8$ and $\max \left(E\left(I_{v}\right) \mp K \sqrt{\operatorname{var}\left(I_{v}\right)}\right)=0.4$ at $t=0$. Here, $K=3$ gives an approximate $99 \%$ confidence interval.

\section{Stochastic model}

The deterministic system (1) will similarly be used to obtain a stochastic model of malaria disease. The randomness in the real life occurences of malaria will be modeled by using stochastic noise in the system of stochastic differential equations. The stochastic effect terms include Wiener processes, which are also known as Brownian Motion. The stochastic model is defined as.

$$
\begin{aligned}
& d S_{h}=\left(\mu(1-\pi)-\sigma S_{h}-\eta S_{h} I_{v}+k I_{h}+\left(1-I_{h}\right) \varphi\right) d t+\Psi_{1} S_{h} d W_{1} \\
& d I_{h}=\left(\eta S_{h} I_{v}-(k+m) I_{h}\right) d t+\Psi_{2} I_{h} d W_{2} \\
& d I_{v}=\left(I_{h}\left(1-I_{v}\right)-\omega I_{v}\right) d t+\Psi_{3} I_{v} d W_{3}
\end{aligned}
$$

along with the same initial conditions: $S_{h}(0)=0.5, I_{h}(0)=0.5, I_{v}(0)=0.4$. Here the Wiener processes $W_{1}, W_{2}$ and $W_{3}$ are independent and the diffusion coefficients $\Psi_{1}, \Psi_{2}$ and $\Psi_{3}$ are also independent coefficients. System (3) contains nonlinear stochastic differential equations, hence like the deterministic and random models, its numerical solution will be examined.

\subsection{Stochastic results}

Stochastic Milstein Scheme has been used for obtaining the numerical solutions of the stochastic model (3). All of the realizations of the stochastic variables $S_{h}, I_{h}$ and $I_{v}$ can be given in a single graph to visualize the stochastic behavior of the model similar to Figures 2 and 3 (Figure 7). The extremum points in the stochastic results are as follows: $S_{h}$ gets a

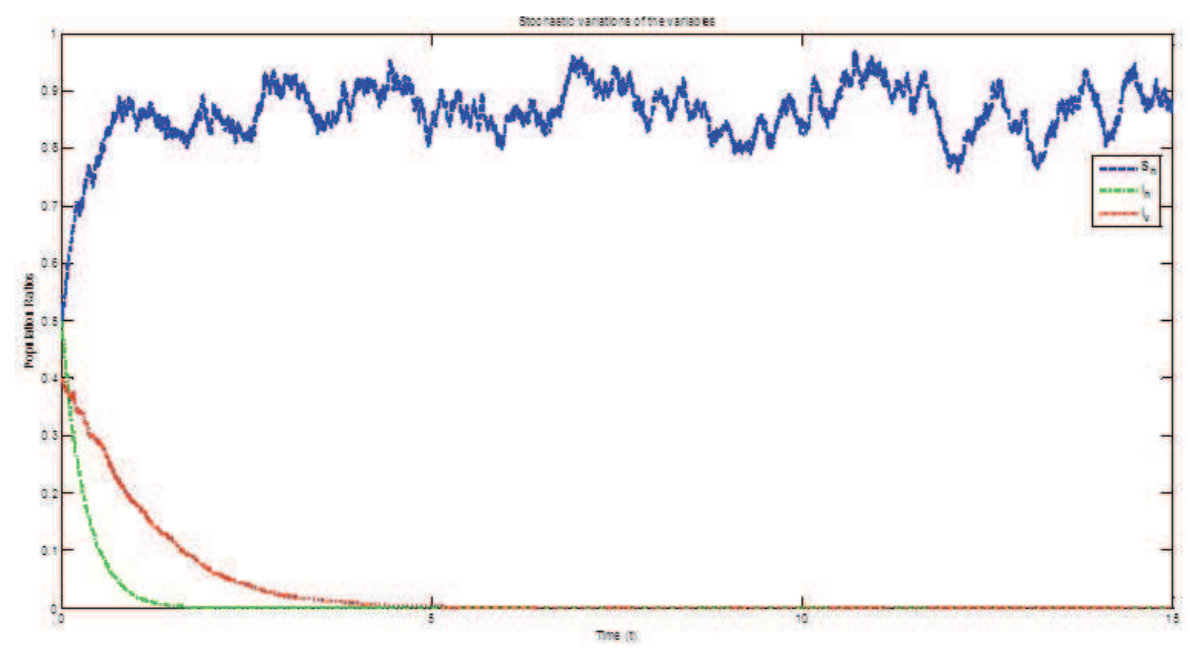

Fig. 7: Realizations of the stochastic variables.

maximum value of 0.968 at $t=10.71$ and a minimum value of 0.5 at $t=0 . I_{h}$ gets a maximum value of 0.5 at $t=0$ and a 
minimum value of $1.647 \times 10^{-10}$ at $t=15 . I_{v}$ gets a maximum value of 0.4 at $t=0$ and a minimum value of $7.931 \times 10^{-8}$ at $t=14.99$. The stochastic results were obtained for a very small amount of stochastic effect with the diffusion coefficient being only 0.1 .

The realizations of the stochastic variables $S_{h}, I_{h}$ and $I_{v}$ have been found as below (Figure 8).
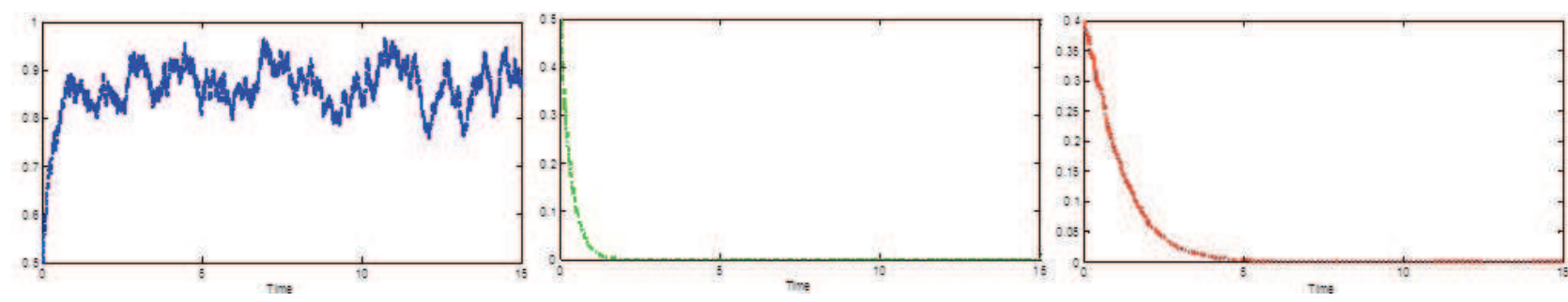

Fig. 8: Realizations of the variables $S_{h}, I_{h}$ and $I_{v}$, respectively.

\section{Comparison of the results}

Minimum and Maximum values obtained in the realizations of the model (3) are given in Table 1. Figures 1, 3 and 7 show

Table 1: Extremum values in deterministic, random and stochastic models.

\begin{tabular}{|l|l|l|l|l|l|l|}
\hline & Deterministic Max & Random Max & Stochastic Max & Deterministic Min & Random Min & Stochastic Min \\
\hline$S_{h}$ & $(0.874,1.554)$ & $(0.8764,1.65)$ & $(0.968,10.71)$ & $(0.5,0)$ & $(0.5,0)$ & $(0.5,0)$ \\
\hline$I_{h}$ & $(0.5,0)$ & $(0.5,0)$ & $(0.5,0)$ & $\left(2.171 \times 10^{-10}, 15\right)$ & $\left(4.495 \times 10^{-10}, 15\right)$ & $\left(1.647 \times 10^{-10}, 15\right)$ \\
\hline$I_{v}$ & $(0.4,0)$ & $(0.4,0)$ & $(0.4,0)$ & $\left(1.114 \times 10^{-7}, 15\right)$ & $\left(2.324 \times 10^{-7}, 15\right)$ & $\left(0.7931 \times 10^{-7}, 14.99\right)$ \\
\hline
\end{tabular}

that the behavior of the components $S_{h}, I_{h}$ and $I_{v}$ are similar in all models, hence the results are meaningful. Note that the stochastic results are from a single realization of the event and may vary in other trials.

\section{Conclusions}

Equation system (1) is a nondimensionalized version of a 5-equation system given in [13]. The 5-equation system describes the changes in the equations $S_{h}, I_{h}$ and $I_{v}$ along with $R_{h}$ and $S_{v}$ where $R_{h}$ is the ratio of recovered humans and $S_{v}$ is the ratio of susceptible mosquitoes. It is known that $S_{h}+I_{h}+R_{h}=1$ for the human population. Hence, from by the expected value operator properties, we can write $E\left(S_{h}\right)+E\left(I_{h}\right)+E\left(R_{h}\right)=1$. Using the result above, since $E\left(S_{h}(t)\right)=0.8752$ and $E\left(I_{h}(t)\right)=4.495 \times 10^{-10}$ at the end of the process, we find that $E\left(R_{h}\right) \simeq 0.12$ at $t=15$. Thus we can say that about $12 \%$ of the whole population can be expected to be recovered from the disease at $t=15$, while about $88 \%$ of the population can be expected to stay susceptible to the disease at the end of the process. The disease is expected to be almost extinct from the human population at about $t=1.5$, as seen from the graph (Figure 3).

Another point that should be noted is the high level of randomness in the variable $S_{h}$. Both the graphs for the confidence interval of $S_{h}$ and the realization of the stochastic variable show that a significant difference can occur between the deterministic results and the random realization of the varaible in real life. $\max \left[\operatorname{var}\left(S_{h}\right)\right]=0.002896$ is the maximum value of the variance of the variable $S_{h}$ meaning that the standard deviance of this variable will become 
$\sqrt{\operatorname{Var}}=\sqrt{0.002896} \simeq 0.054$. This means that the real life random occurrence of the value of $S_{h}$ could be expected to be about $5.4 \%$ greater or less than what the deterministic model suggests. This level of variation is a point that can not be discarded in a disease model and shows how important random modeling and stochastic modeling studies can be on this field.

\section{Discussion}

The deterministic model given in [13] was added random effects and stochastic noise to obtain the random (2) and the stochastic models (3). Numerical results for both models were compared with each other and the results of the deterministic model. The results show that both models are meaningful and that their results are in accordance with the deterministic model. The results also show that the random and stochastic models describe the random nature of the model components and provide useful information on the randomness of the event. The random analysis of Malaria can be improved by investigating the real life data for the exact distribution of the random values for various parameters of the model. The stochastic model could also be analyzed in more detail by using other schemes for the numerical solutions and making a comparison of the stochastic results as well.

\section{Competing interests}

The authors declare that they have no competing interests.

\section{Authors' contributions}

All authors have contributed to all parts of the article. All authors read and approved the final manuscript.

\section{References}

[1] Aron, J.L. (1989). Mathematical modelling of immunity to malaria. Math. Biosci., 90, 385-396.

[2] Anderson, R.M. \& May, R.M. (1991). Infectious Diseases of Humans: Dynamics and Control, Oxford University Press, Oxford.

[3] Bailey, N.T.J. (1988). The Biomathematics of Malaria. In: Malaria: Principles and Practice of Malariology, Oxford Uni. Press, London.

[4] Bekiryazici, Z., Merdan, M., Kesemen, T. \& Najmuldeen, M. (2016). Mathematical Modeling of Dengue Disease under Random Effects. Mathematical Sciences and Applications E-Notes, 4(2), 58-70.

[5] Caraballo, H. (2014). Emergency department management of mosquito-borne illness: Malaria, dengue, and west nile virus. Emergency Medicine Practice, 16(5), 1-23.

[6] Chiyaka, C., Garira, W. \& Dube, S. (2007). Transmission model of endemic human malaria in a partially immune population. Mathematical and Computer Modelling, 46, 806-822.

[7] Feller, W. (1971). An Introduction to Probability Theory and Its Applications, vol. 2, John Wiley \& Sons, New York.

[8] Gaudart, J., Touré, O., Dessay, N., Dicko, A.I., Ranque, S., Forest, L., Demongeot, J. \& Doumbo, O.K. (2009). Modelling malaria incidence with environmental dependency in a locality of Sudanese savannah area, Mali, Malaria Journal, 8(61).

[9] Gerardin, J., Ouédraogo, A.L., McCarthy, K.A., Eckhoff, P.A. \& Wenger, E.A. (2009). Characterization of the infectious reservoir of malaria with an agent-based model calibrated to age-stratified parasite densities and infectiousness, Malaria Journal, 14(231).

[10] Gurarie, D. \& McKenzie, F.E. (2007). A stochastic model of immune-modulated malaria infection and disease in children, Math Biosci, 210(2), 576-597.

[11] Merdan, M. \& Khaniyev, T. (2008). On the Behavior of Solutions under the Influence of Stochastic Effect of Avian-Human Influenza Epidemic Model, International Journal of Biotechnology and Biochemistry, 4(1), 75-100. 
[12] Ngwa, G.A. \& Shu, W.S. (2000). A Mathematical Model for Endemic Malaria with Variable Human and Mosquito Population, Mathematical and Computer Modelling, 32, 747-763.

[13] Nirwani, N., Badshah, V.D. \& Khandewal, R. (2015). A Mathematical Model of Malaria Disease with Vertical Transmission. Journal of Mathematics Research, 7(3), 159-164.

[14] Smith, T.A. (2008). Estimation of heterogeneity in malaria transmission by stochastic modelling of apparent deviations from mass action kinetics. Malaria Journal, 7(12).

[15] World Health Organization (2015). Fact Sheet: World Malaria Report 2015.

[16] Yang, H.M. (2000). Malaria transmission model for different levels of acquired immunity and temperature dependent parameters (vector). J Public Health, 34, 223-231. 